\title{
REFINEMENTS OF KY FAN'S EIGENVALUE INEQUALITY FOR SIMPLE EUCLIDEAN JORDAN ALGEBRAS BY USING GRADIENTS OF $K$-INCREASING FUNCTIONS
}

\author{
MAREK NiEZGODA
}

Abstract. In this paper, by using some analytical methods based on gradients of $K$-increasing functions, we show refinements of the following Ky Fan like inequality: $\lambda(x+y) \prec \lambda(x)+\lambda(y)$ with elements $x$ and $y$ of a simple Euclidean Jordan algebra, the eigenvalue operator $\lambda(\cdot)$ and Schur's majorization $\prec$.

Mathematics subject classification (2020): 17C50, 26B25, 26D15, 15A18.

Keywords and phrases: Majorization, Ky Fan's eigenvalue inequality, simple Euclidean Jordan algebra, $K$-increasing function, gradient.

\section{REFERENCES}

[1] R. Bhatia, Matrix Analysis, Springer-Verlag, New York, 1997.

[2] K. FAN, On a theorem of Weyl concerning eigenvalues of linear transformations I, Proc. Nat. Acad. Sci. U.S.A., 35, (1949), 652-655.

[3] K. FAN, Maximum properties and inequalities for the eigenvalues of completely continuous operators, Proc. Nat. Acad. Sci. U.S.A., 37, (1951), 760-766.

[4] L. Fay bus ovich, Several Jordan-algebraic aspects of optimization, Optimization, 57, (2008), 379393.

[5] A. S. LEwIS, Convex analysis on Cartan subspaces, Nonlinear Anal., 42, (2000), 813-820.

[6] Y. Lim, J. Kim AND L. FAYBusovich, Simultaneous diagonalization on simple Euclidean Jordan algebras and its applications, Forum Math., 15, (2003), 639-644.

[7] L. Maligranda, Simple norm inequalities, Amer. Math. Monthly, 113, 3 (2006), 256-260.

[8] A. W. Marshall, I. Olkin AND B. C. ARnold, Inequalities: Theory of Majorization and Its Applications, 2nd Ed., Springer, New York, 2011.

[9] M. M. Moldovan, A Gersgorin type theorem, special inequalities, and simultaneous stability in Euclidean Jordan algebras, PhD Thesis, University of Maryland Baltimore County, 2009.

[10] M. NiEZGodA, An extension of Schur-Ostrowski's condition, weak Eaton triples and generalized AI functions, Linear Algebra Appl., 580, (2019), 212-235.

[11] M. NieZgodA, On Maligranda like inequalities for G-majorization, Linear and Multilinear Algebra, published online, 27 I 2020, doi:10.1080/03081087.2020.1720576.

[12] M. NiEZGODA, On triangle inequality for Miranda-Thompson's majorization and gradients of increasing functions, Adv. Oper. Theory, 5, (2020), 647-656.

[13] M. NiEZGODA, Refinements of triangle like inequalities in Lie's framework, Bull. Malays. Math. Sci. Soc., 44, 1 (2021), 243-250.

[14] M. NiEZgODA AND T.-Y. TAM, On the norm property of $G(c)$-radii and Eaton triples, Linear Algebra Appl., 336, (2001), 119-130.

[15] J. TAO, L. Kong, Z. Luo And N. XiU, Some majorization inequalities in Euclidean Jordan algebras, Linear Algebra Appl., 461, (2014), 92-122. 